LBNL-52235

\title{
Investigation of Residential Central Air Conditioning Load Shapes in NEMS
}

\author{
Prepared for the \\ Office of Building Technologies \\ Assistant Secretary for Energy Efficiency and Renewable Energy \\ U.S. Department of Energy \\ Principal Authors \\ Kristina Hamachi LaCommare, Chris Marnay, Etan Gumerman, Peter Chan, \\ Greg Rosenquist, and Julie Osborn \\ Ernest Orlando Lawrence Berkeley National Laboratory \\ 1 Cyclotron Road, MS 90-4000 \\ Berkeley CA 94720-8061
}

May 2002

This work described in this paper was funded by the Assistant Secretary of Energy Efficiency and Renewable Energy, Office of Building Technologies of the U.S. Department of Energy under Contract No. DE-AC03-76SF00098 



\section{Table of Contents}

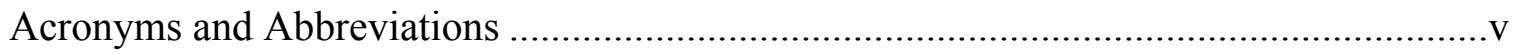

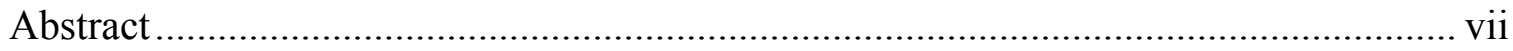

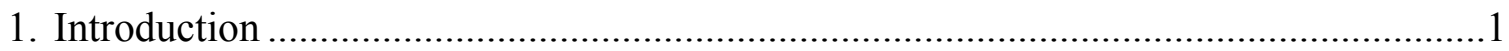

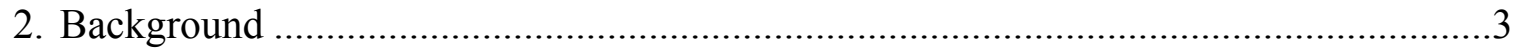

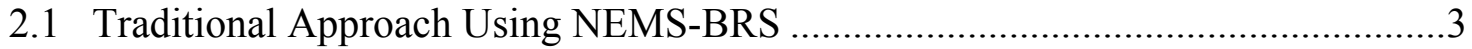

2.2 Details of the End-Use Decrement Methodology ..................................................

2.3 What is the Conservation Load Factor? .............................................................4

3. Input Load Shape Structure in NEMS-BRS .........................................................

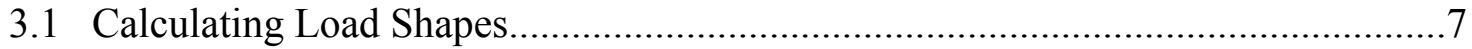

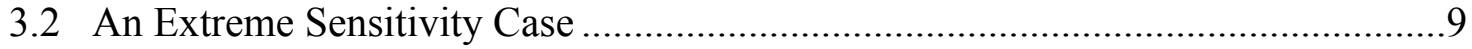

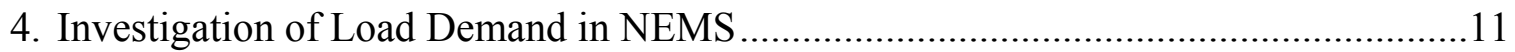

4.1 Traditional vs. Delta Approach..........................................................................11

4.2 Complexity of the Delta Approach .................................................................. 12

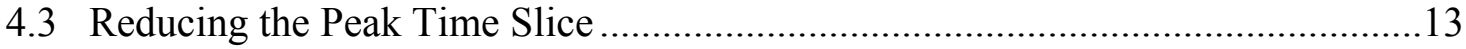

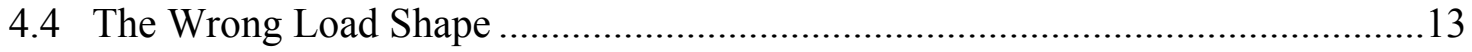

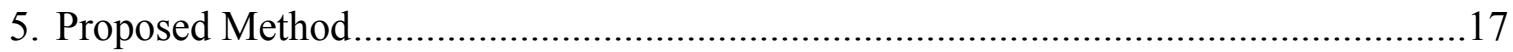

5.1 The Efficiency-Modifying Approach .............................................................17

5.2 The 18-point Approach ................................................................................ 18

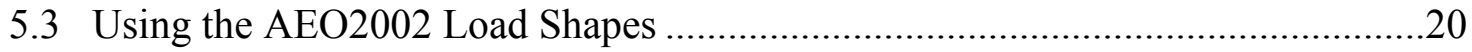

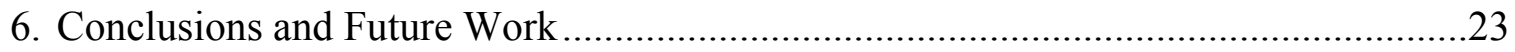

Appendix A: Mathematical Representation of Load Demand in NEMS .......................25

Appendix B: Detailing the Traditional End-Use Decrement Approach Using the 2002 Load

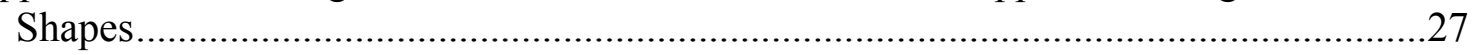





\section{List of Figures and Tables}

Figure 1. August Hourly Load for U.S. Residential CAC from AEO2001 ……........................... 9

Figure 2. Change in Other Fossil Capacity for 18 Runs ........................................................... 19

Figure 3. NOx Marginal Emissions Factors from 18 Runs (2015)............................................ 20

Table 1. CLFs based on Historic Studies .................................................................................... 5

Table 2. Comparing the CLF Using 2 Different CAC Load Shapes Under the APS Customary Energy Decrement Approach in AEO2000 and AEO2001 …………………………..... 14

Table 3. Comparing 2020 CLFs from Various Approaches Using TSL 4 .................................. 21 



\section{Acronyms and Abbreviations}

$\begin{array}{ll}\text { AEO } & \text { Annual Energy Outlook } \\ \text { APS } & \text { appliance energy efficiency standards } \\ \text { BRS } & \text { Department of Energy's Building and Research Standards program } \\ \text { CAC } & \text { central air conditioning } \\ \text { CLF } & \text { conservation load factor } \\ \text { DOE } & \text { U.S. Department of Energy } \\ \text { EIA } & \text { Energy Information Administration } \\ \text { FSEC } & \text { Florida Solar Energy Center } \\ \text { GW } & 10^{9} \text { (giga)watt } \\ \text { HP } & \text { heat pump } \\ \text { kW } & 10^{3} \text { (kilo)watt } \\ \text { LDC } & \text { load duration curve } \\ \text { LDSM } & \text { Load and Demand Side Management submodule } \\ \text { MEF } & \text { marginal emissions factor } \\ \text { NAECA } & \text { National Appliance Energy Conservation Act of 1987 } \\ \text { NEMS } & \text { National Energy Modeling System } \\ \text { NERC } & \text { North American Reliability Council } \\ \text { NES } & \text { National Energy Savings model } \\ \text { PG\&E } & \text { Pacific Gas \& Electric } \\ \text { RECS } & \text { Residential Energy Consumption Survey } \\ \text { SCE } & \text { Southern California Edison } \\ \text { SEER } & \text { seasonal energy efficiency ratio } \\ \text { TSL } & \text { trial standard level } \\ \text { TWh } & 10^{12} \text { (tera)watt hour }\end{array}$





\begin{abstract}
This memo explains what Berkeley Lab has learned about how the residential central airconditioning (CAC) end use is represented in the National Energy Modeling System (NEMS). NEMS is an energy model maintained by the Energy Information Administration (EIA) that is routinely used in analysis of energy efficiency standards for residential appliances. As part of analyzing utility and environmental impacts related to the federal rulemaking for residential CAC, lower-than-expected peak utility results prompted Berkeley Lab to investigate the input load shapes that characterize the peaky $\mathrm{CAC}$ end use and the submodule that treats load demand response. Investigations enabled a thorough understanding of the methodology by which hourly load profiles are input to the model and how the model is structured to respond to peak demand. Notably, it was discovered that NEMS was using an October-peaking load shape to represent residential space cooling, which suppressed peak effects to levels lower than expected. An apparent scaling down of the annual load within the load-demand submodule was found, another significant suppressor of the peak impacts.
\end{abstract}

EIA promptly responded to Berkeley Lab's discoveries by updating numerous load shapes for the AEO2002 version of NEMS; EIA is still studying the scaling issue.

As a result of this work, it was concluded that Berkeley Lab's customary end-use decrement approach was the most defensible way for Berkeley Lab to perform the recent CAC utility impact analysis. This approach was applied in conjunction with the updated AEO2002 load shapes to perform last year's published rulemaking analysis. Berkeley Lab experimented with several alternative approaches, including modifying the CAC efficiency level, but determined that these did not sufficiently improve the robustness of the method or results to warrant their implementation. Work in this area will continue in preparation for upcoming rulemakings for the other peak coincident end uses, commercial air conditioning and distribution transformers. 



\section{Introduction}

Berkeley Lab has been studying how the National Energy Modeling System (NEMS), a midrange forecast energy model maintained by the Energy Information Administration (EIA), treats and forecasts the peak characteristics of residential central air conditioning (CAC). For more than two years, the Berkeley Lab team of Kristina Hamachi LaCommare, Chris Marnay, Etan Gumerman, Peter Chan, and Julie Osborn have worked to understand how NEMS treats the peak-load reduction from proposed residential CAC energy-efficiency standards. The impetus for this work arose from the NEMS-based utility and environmental analyses for the federal energy efficiency standard rulemaking on residential CAC, which is required by the National Appliance Energy Conservation Act (NAECA) of 1987. This project was the first rulemaking analysis Berkeley Lab performed for a peak-load-coincident end use. When peak-load reductions from this analysis were significantly lower than expected using the customary end-use decrement approach, it became important to understand why the model was apparently deflating the expected peak impacts. So Berkeley Lab began an intensive search to uncover the mystery behind the CAC load shape structure and its treatment in NEMS.

The subsections below explain what Berkeley Lab has discovered regarding NEMS's treatment of CAC loads:

- Section 2 provides background information to explain the traditional end-use decrement approach Berkeley Lab has established as the conventional method for performing utility and environmental impact analyses for residential and commercial appliance standards rulemakings. This section includes an explanation of the conservation load factor (CLF), which is a possible measure of how well NEMS translates energy savings from the peaky CAC end use to peak load savings.

- Section 3 describes what Berkeley Lab has learned about the detailed structure of the NEMS input load shape file. This section explains the temporal characteristics of this large input file and the method NEMS uses to construct an hourly $\mathrm{kW}$ profile based on scalar energy use input parameters.

- Section 4 explains NEMS coding in detail, looking at how the Load and Demand Side Management (LDSM) submodule treats input load profiles and calculates the net load reduction resulting from a proposed residential CAC standard. It was discovered that the residential CAC load shape in NEMS is represented by a late autumn-peaking load shape that distorts representation of the cooling load, which is highest in summer. NEMS also contains a piece of code (nicknamed the squelch) that appeared to be scaling down system loads to match historical trends.

- Section 5 presents some of the approaches that Berkeley Lab explored to improve the NEMS analysis, including modifying the efficiency level of the $\mathrm{CAC}$ end use and experimenting with a larger sample size to perform a linear regression for the utility-sector analysis.

- Section 6 summarizes what Berkeley Lab has learned on this subject and presents conclusions and some possible next steps for future work. 



\section{Background}

\subsection{Traditional Approach Using NEMS-BRS}

Berkeley Lab uses the NEMS-BRS model to forecast the energy impacts that proposed appliance standards will have on the power sector and the environment through the year 2020. Berkeley Lab calls the version of NEMS used to model proposed energy-efficiency standards NEMS-BRS because this work is conducted under the auspices of the Department of Energy (DOE) Building and Research Standards (BRS) program. EIA requires that any modified version of NEMS be named differently, to distinguish them from EIA's Annual Energy Outlook (AEO) Reference Case version of NEMS. Throughout this memo, NEMS-BRS is used to refer to the appliance standards' modified version of NEMS used at Berkeley Lab. The AEO version of the model is referred to as simply NEMS.

The NEMS-BRS analysis reports changes resulting from varying appliance standards; these include changes in installed generating capacity and the corresponding equivalent number of power plants avoided, electric generation saved by the power sector, and carbon and NOx emissions saved by electricity generators.

The standard approach to analyzing a proposed appliance standard in the residential or commercial sector is to model the impacts of the standard by reducing the end-use consumption for each modeled year organized by regional census divisions; this subtracted value which is calculated exogenously to NEMS-BRS is referred to as the energy decrement. This approach worked well for most end uses previously studied (residential clothes washers, water heaters, and commercial fluorescent ballasts); however, the CAC proposed rulemaking raised concerns because NEMS-BRS did not appear to be accurately reflecting the amount of installed generating capacity potentially saved in the utility sector. Before the concerns raised by the CAC analysis are discussed, the next subsection describes the detailed steps involved in the standard analysis of appliance standards.

\subsection{Details of the End-Use Decrement Methodology}

This portion of the memo explains the process of deriving utility impacts and environmental emissions savings for a proposed appliance standard using the established end-use decrement approach. The paragraphs below address how relatively small energy savings from a residential end use can be modeled in a large multi-sectoral national energy sector model to derive results that are clearly distinct from energy-sector-scale fluctuations.

Because the relative size of appliance energy efficiency standard (APS) savings implemented in NEMS-BRS is so small compared to the fluctuations in energy use by the whole energy sector, multiple NEMS-BRS runs must be performed using larger decrements that are extrapolated from the small, proposed energy savings. Interpolated values for the NEMS-BRS savings are derived from this series of larger-decrement simulations; the savings in each of the runs are based on an appliance energy efficiency standard trial standard level (TSL), which represents a certain combination of energy efficiency improvements to the appliance that are considered technically feasible by Berkeley Lab engineers. 
The electricity generation and capacity output by fuel type for each of the NEMS-BRS iterations (i.e., 4, 6, and 8 times the TSL) are then regressed, and the actual forecast of the impact of the standard is interpolated back to the Reference Case at the origin. The linear regression is forced through the origin because a zero change will result if no standard is enacted.

The estimated reduction in thermal generation from each TSL as determined by interpolation is then used to determine emissions savings. First, annual marginal emissions rates are calculated for each of the simulations in a savings group, based on the output from NEMS-BRS. Marginal emissions rates incorporate two effects of a standard: the emissions saved by the reduction in total generation and the change in the emissions characteristics of the whole power sector that result from the slowing of new plant construction. Experience shows that the net effect of an appliance standard on the system is very small, and the overall effect on emissions can be fully attributed to the decrement in generation. The annual marginal emission rates at the TSL are then extrapolated from these rates (at multipliers of the TSL savings) by taking a simple average.

The constant emission rate that emerges is found at higher decrement levels and is assumed to hold for the range of small decrements that correspond to the various standards. For all years up to the final forecast year (2020), marginal emission rates are calculated by averaging the marginal rates of the three higher decrement runs. Total emissions savings in each year are the product of the annual marginal emission rate and the reduction in total generation for that year (as calculated by the interpolation method described above).

The methodology described above was used for the utility and environmental analysis related to the federal rulemakings for residential clothes washers, water heaters, and commercial fluorescent ballasts. However, unlike these other end uses, the residential CAC end use is peaky, and lower-than-expected peak impacts raised concerns about how NEMS was treating cooling.

\subsection{What is the Conservation Load Factor?}

Berkeley Lab has been performing the utility and environmental analyses of energy-efficiency standards' rulemaking using NEMS-BRS for several years. Residential CAC was the first peaky end use for which residential energy-efficiency standards were proposed and this work grew from a subsequent rulemaking. Analysis of non-peaky end uses is simpler because their powersector impacts are not strongly coincident with peak demand times, so the impact of a standard on new power plant construction is less significant. The residential CAC end use, however, is characteristically peaky, and the analysis of the proposed TSL for this end use in the residential sector has raised concerns because the power sector impacts were generally lower than expected considering the heavy use of CAC during high-demand or peak periods.

The conservation load factor (CLF) serves as a measure of how peaky an end use is. The CLF is the ratio of the average year-round load savings to the peak load savings. For an end use like refrigerators, whose baseload shape is flat, a higher CLF value of approximately 60 to $70 \%$ is typical. This value indicates that average load of the end use is 60 to $70 \%$ of its peak load, yielding a flatter (baseload) profile. However, for a peaky end use like CAC, the CLF is 
typically in the range of $10-20 \%{ }^{1}$ The high-demand episodes are largely on hot, summer days when CAC use is high. Because CAC is a peak-coincident end use, a CAC standard will result in large peak demand savings relative to the energy saved. For example, using the established end-use decrement approach, the proposed TSL 4 (equivalent to an seasonal energy efficiency ratio, or SEER, of 13), which saves an estimated 36.5 TWh of site energy in 2020, results in a reduction of only $11.7 \mathrm{GW}$ of installed generating capacity, translating to a CLF of roughly $46 \%$. That is, the peak reduction result from AEO2001 is at least 10 to $20 \mathrm{GW}$ lower than expected and the corresponding CLF is substantially higher. Capacity changes should be closer to $25 \mathrm{GW}$ with a typical CLF of $15 \%$.

Because of this discrepancy, Berkeley Lab looked into exogenous case studies of CAC use in various geographic locations to calculate CLFs based on annual average usage and peak demand. Table 1 below presents estimated CLFs from case studies on AC use in locations across the United States. The Southern California Edison (SCE) and Pacific Gas and Electric (PG\&E) case studies focus on their respective service territories within California; CLFs are less than 10\% in both cases. The Florida Solar Energy Center (FSEC), the only other study with sufficient data, yielded a CLF of $12.7 \%$, more along the lines of what was expected for this type of end use. The U.S. national averaged reported by Koomey et al of 15\% illustrates the variability apparent in regional versus nationally averaged CLFs. In general, this table supports the conventional wisdom that residential CAC use is characteristically peaky with a low CLF.

Table 1. CLFs based on Historic Studies

\begin{tabular}{|l|c|c|}
\hline Source & CLF & Location \\
\hline SoCal Ed 1991 & $8.34 \%$ & SCE district \\
\hline PG\&E Amp 1992: & & \\
Zone R & $7.26 \%$ & Desert/Mtn - very hot \\
Zone S & $6.95 \%$ & Valley - hot \\
Zone X & $3.32 \%$ & Hills - moderate climate \\
\hline UPA 1992 & & Minnesota \\
\hline FSEC 1996 & $12.7 \%$ & Homestead, FL (10 homes) \\
\hline & & U.S. National Average \\
\hline Koomey et al. ${ }^{1}$ & $15.0 \%$ & . \\
'Source: Koomey et al., Conservation screening curves to compare efficiency \\
investments to power plants, Energy Policy, Volume 18 No. 8, October 1990.
\end{tabular}

Berkeley Lab relied heavily on the CLF in the analysis of how well NEMS-BRS represented the residential CAC end use. A number of approaches were tested to determine the best way to

\footnotetext{
${ }^{1}$ The CLF concept is discussed in more detail in: Koomey, Rosenfeld, and Gadgil. 1989. "Conservation Screening Curves to Compare Efficiency Investments to Power Plants.” Paper from 1990 ACEEE Summer Study Conference proceedings and a paper with same title and authors from Energy Policy, Volume 18 No. 8, October 1990.
} 
Investigation of Residential Central Air Conditioning Load Shapes in NEMS

represent the CAC end use's peak coincidence; the CLF is used to compare how each approach represents CAC's peak coincidence. 


\section{Input Load Shape Structure in NEMS-BRS}

In late 1999 and early 2000 when concern was first raised about CAC utility impact results, Berkeley Lab began to investigate the load shapes in NEMS-BRS. The structure of the load profiles was interpreted, and it was noted that the load shapes are input as percent contributions to the overall load and divided into a series of time slices to represent three day types for each month- peak, weekday, and weekend.

Review of the model and documentation showed that NEMS is structured so that:

1. There is no regional differentiation of load shapes for residential end uses in NEMS versions earlier than AEO2002. In other words, NEMS contains only one load shape for the nation for each end use in versions prior to AEO2002. The ten residential end uses that have unique load shapes are: space cooling, space heating, refrigerators, freezers, water heaters, dryers, cooking, lighting, other appliances, and secondary heating.

2. Each load shape is composed of 864 data points (12 months * 3 day types per month * 24 hourly values for each day type).

3. Although NEMS performs computations for each month, the input values are based on seasonal estimates. For space cooling, the four seasons are December-March, April-May, June-September and October-November.

4. The space cooling load shape includes usage for April-November (5,856 hours) and is zero for the remaining months.

5. Although there are separate heating and cooling load shapes, there are not separate load shapes for CAC versus heat pumps. All cooling load is assigned to the space cooling load shape, and heating load is assigned to the space heating load shape. The heating load shape includes usage for December-March (2,904 hours) and is zero for the remaining months.

6. The load shapes for all end uses are found in the all1lsr input file. This file is preprocessed to create a direct access file that is read in by NEMS. According to John Holte of OnLocation, the load shape data were supplied by EPRI.

7. The ldsmstr input file defines the structure for the Load and Demand-Side Management (LDSM) submodule and contains the pointers indicating which end use corresponds to which load shape in the allilsr file.

\subsection{Calculating Load Shapes}

NEMS determines hourly, end-use loads through a four-step process:

1. The model starts with an annual end-use load energy value, which is based on the annual sales forecast for a region. 
2. The annual end-use load is then divided into monthly sales forecasts based on exogenously input monthly allocation factors (in the all1lsr input file) that vary by end use. For residential space cooling, the monthly allocation factors, which sum to 100, are:

$\begin{array}{llllllllllll}\text { Jan } & \text { Feb } & \text { Mar } & \text { Apr } & \text { May } & \text { Jun } & \text { Jul } & \text { Aug } & \text { Sep } & \text { Oct } & \text { Nov } & \text { Dec } \\ 0.0 & 0.0 & 0.0 & 1.4 & 1.5 & 23.5 & 23.5 & 25.6 & 21.9 & 1.4 & 1.3 & 0.0\end{array}$

3. Monthly loads are then allocated into daily loads with a set of day-type allocation factors that specify the energy use for each day type relative to the peak day value (1.000). Within a month, all days assigned to a given day type are assumed to have the same load (the season/day weights are in the all1lsr input file). For space cooling, the day type allocation factors for months with loads are:

$\begin{array}{lccc}\text { Season } & \text { Weekday } & \text { Weekend } & \text { Peak Day } \\ \text { Apr-May } & 0.0304 & 0.0445 & 1.000 \\ \text { Jun-Sep } & 0.2879 & 0.4290 & 1.000 \\ \text { Oct-Nov } & 0.0304 & 0.0445 & 1.000\end{array}$

These allocation factors are normalized to a peak day value of 1.000. The normalized value is calculated by multiplying the number of days in the month assigned to each day type by the allocation factor for that day type. The daily factor is then divided by the sum of the monthly total to determine the fraction of monthly energy allocated to that day type.

4. Each day's load is then divided into a set of hourly loads for that day. These allocation factors (in the all1lsr input file) are based on season and day type. Like the monthly allocation factors, these factors sum to 100 .

This four-step process enabled a graphical depiction of the $\mathrm{kWh}$ profile for each day type from the weighted nondimensional input parameters. As an example, Figure 1 below illustrates an hourly $\mathrm{kW}$ profile for residential CAC use in the U.S. derived from the 2001 version of NEMS load shape input file. Hourly profiles are shown for each of the three-day types - (peak day, weekday, and weekend) -- for the month of August. 


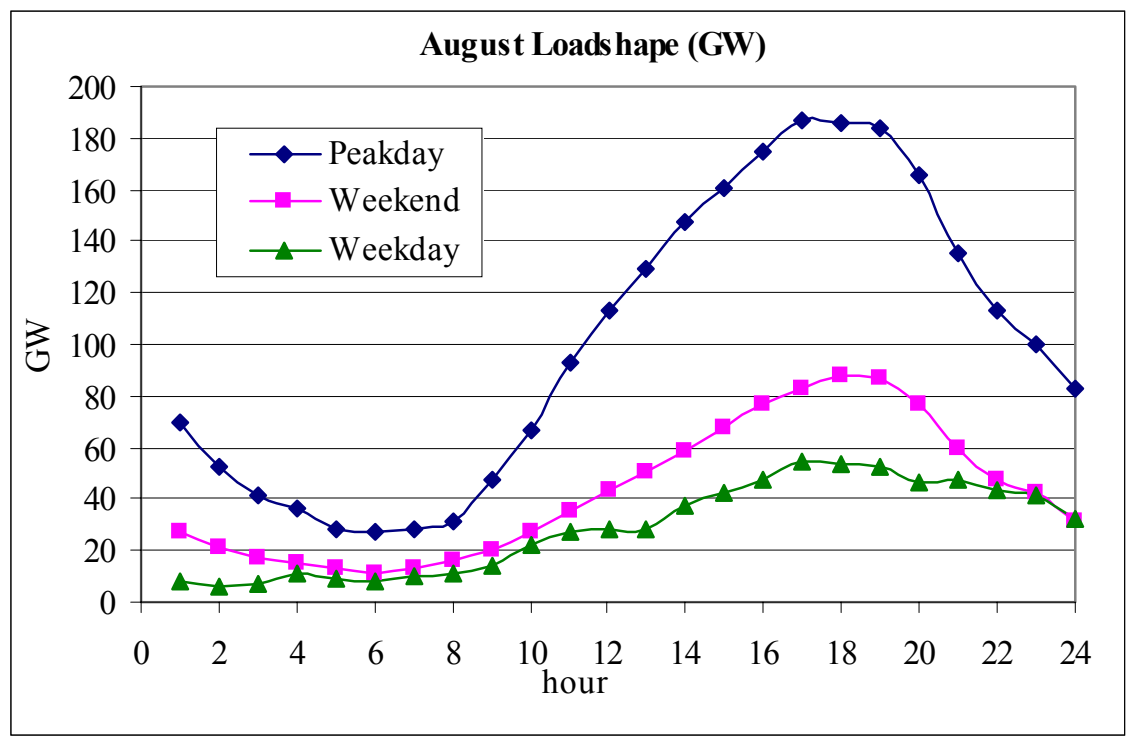

Figure 1. August Hourly Load for U.S. Residential CAC from AEO2001

The next subsection describes Berkeley Lab's effort to test understanding of the load shape structure in NEMS with an experiment that shifted the input load profile to represent a characteristically peaky end use.

\subsection{An Extreme Sensitivity Case}

As an exercise, Berkeley Lab discovered that shifting virtually all the CAC load to the peak day did not produce the dramatic effects that would logically result. The paragraphs below describe this experiment and its results.

Almost all the CAC load was shifted to the peak day in this exercise, removing the demand from all shoulder non-peak hours. This experiment focused on two residential CAC load shapes in the all1lsr file, which were preprocessed to make the ldsmdaf input file. The two modified end uses chosen for this exercise are RSFCAEW7 and RSFCA067. The RSFCAEW7 load profile was redefined to have 100\% demand during August, more than $99 \%$ of which was on the peak day between 7 a.m. and 7 p.m. RSFCAO67 was redefined to have about $23 \%$ of demand in each of the summer months, June-September. July and August demand was concentrated to $94 \%$ on peak days.

Surprisingly, this extreme sensitivity case resulted in only minimal load demand response impacts. The reason for this was later determined to be that the RSFCAO67 load shape is one of many in the all1lsr input file that is not used in the model. This finding is discussed in further detail in the next section. In addition to the fact that this file is not used, there is another important explanation for the lack of impact in this experiment. Simply shifting the fraction of annual demand to the peak day isn't necessarily an extreme sensitivity case in NEMS because the peak demand periods for some end uses do not coincide with the system peak. Although this exercise did not accomplish the intended goal of determining the effect of shifting the load, it did raise the question of whether the analyzed NEMS load shapes were even being used. 
Investigation of Residential Central Air Conditioning Load Shapes in NEMS

The next section describes how the NEMS coding was scrutinized to understand what happens to the CAC load shapes once input to the model. 


\section{Investigation of Load Demand in NEMS}

This section presents the mathematical and conceptual details of how load demand is treated in NEMS. One of the key discoveries was the presence of the Delta Approach for calculating the system load, which is designed to ensure that the system load matches the historical system load shape. This method is used by NEMS even though the model also includes a disabled bottom-up approach. Using intermediary output data, Berkeley Lab determined that these two different approaches to calculate forecast peak demand yield very different CLFs, which could explain why the peak impacts from a proposed CAC TSL are so apparently inaccurate. Another interesting discovery was a piece of code that appeared to be scaling down the annual system load to match the historical data trend. Without the scaling factor, the Reference Case system load increased by approximately $38 \mathrm{GW}$ or $3.5 \%$ in 2020 . The authors have dubbed this effect the squelch.

The following subsections detail the findings regarding load demand in NEMS. Section 4.1 presents the discovery of the two approaches (traditional and Delta) for forecasting system load in NEMS. Section 4.2 describes the two approaches within the Delta approach, one called the Original Delta Approach, which we deactivated, and the other called the Current Delta Approach, which is currently used in NEMS. An explanation of why EIA chose the Current Delta Approach over the Original Delta Approach is presented in the next section. As well, a mathematical comparison of these approaches is presented in Appendix A. Section 4.3 describes the effect of reducing the peak time period in NEMS from the default $5 \%$ down to $1 \%$ of all hours in the year. Section 4.4 then describes Berkeley Lab's discovery of an October-peaking load profile that EIA assigned to represent the space-cooling end use.

\subsection{Traditional vs. Delta Approach}

Our research revealed that two approaches to generate the system load shape are presented in the LDSM submodule, but only one is used.

The first, the traditional approach, is a bottom-up approach, which constructs the system load shape as the sum of the hourly loads of all end uses. Because this load shape is directly based on the hourly loads of end uses, the subtotals by sector (residential, commercial, transportation, and industrial) can be readily calculated by referring to the sector to which each end use belongs. This bottom-up approach, carrying the impacts from each end use up to the system level, appears to be the most realistic way to determine end-use impacts on the system load. However, this approach is not currently used in NEMS. EIA reports that they were never able to equilibrate forecasted years, so they abandoned this approach. ${ }^{2}$

The other method of calculating the system load shape, which is currently used in NEMS, is called the Delta Approach. According to the NEMS documentation, the Delta Approach takes advantage of the initial system data base and still produces reasonable forecasts of the system load shape. The Delta Approach is intended to ensure that the calculated system load shapes

\footnotetext{
${ }^{2}$ For more information on load demand in NEMS, please refer to the U.S. Department of Energy, Energy Information Administration documentation., Model Documentation: Load and Demand Side Management Submodule, Contract No. DEAC01-92E121946, 1995.
} 
match the initial historic system load shape. Rather than summing all end uses to derive the system load, the Delta Approach scales down the end-use impacts to match historical trends, starting at the system level and working down. This approach ensures that the system load shape will never deviate substantially from historical trends. While this approach solves the problem of mismatched historical estimated and observed system loads, it potentially creates a second problem, namely that changes in enduse energy usage may not get properly translated into electrical load shape changes. The authors' observation that the CLFs calculated by NEMS-BRS do not match expectations based on load shape inputs are a symptom of the problem, but do not show its full extent.

Berkeley Lab was able to write out some peak and system load data from the LDSM as part of the ldsmrpt intermediary file. Using this data set it was possible to analyze the sectoral demand and peak by NERC region for each year as calculated by the traditional bottom-up approach. These outputs also included the total system annual load demand and system peak demand by NERC region for each year as calculated by the Delta Approach. The sectoral annual load and sectoral peak demand of the AEO2001 Reference Case yield a CLF for the residential sector of $14 \%$, and a CLF for the total system of $24 \%$ in 2020 . However, from the annual system demand and system peak calculated by the Delta Approach, the CLF in year 2020 is $41 \%$. The latter is consistent with the prior estimate of the CLF, using the difference in generating capacity as a substitute for the difference in peak demand, which was previously presented as $35 \%$. Thus, these two different approaches to calculate forecasted peak demand yield very different CLFs, which could explain why the peak impacts from a proposed CAC TSL are so apparently underrepresented. The next subsection presents further investigation of the Delta Approach and the scaling factor used to match the historical trends.

\subsection{Complexity of the Delta Approach}

Another peculiarity to the Delta Approach is the presence of two options in the model; one is called the Original Delta Approach and the other is called the Current Delta Approach. Appendix A describes in detail the mathematical representation and differences between the two. These two code segments appear in the LDSM as part of the delta approach for forecasting annual changes in system load. The difference is due to the presence of a scaling factor in the Current Delta Approach. One approach uses this scaling parameter; the other, deactivated or commented out version, uses a similar function but without the scaling factor.

With both Delta Approaches, the system load shape seems to conform to the initial historic system load shape until year 2001. It is not clear how EIA justified switching to the current Delta Approach. As mentioned earlier, reactivating the piece of code corresponding to the original Delta Approach without any other modifications to NEMS results in a 38-GW increase in installed generating capacity in 2020 over the AEO2001 version of NEMS. The system-loadbased CLF, however, drops to $26 \%$ from $41 \%$ in year 2020. This CLF is consistent with the observation demonstrated in Appendix A that the term B cancels out when calculating the difference in the original Delta Approach. Thus, the difference in peak should be similar to that in the bottom-up approach. As mentioned earlier, the CLF from the difference in the bottom-up approach (in the sectoral analysis) was $24 \% .^{2}$ 
These results lead to the conclusion that the current Delta Approach in the NEMS-BRS module, which tries to force annual system load shapes to conform to a rather flat historic load shape, is squelching the peak impact and causing the CLF result to be high. Results with and without the squelch in place are discussed briefly in Section 4.4 .

\subsection{Reducing the Peak Time Slice}

Another exercise looked at a suggestion EIA made to try and reduce the peak time slice or period in NEMS from the default 5\% down to $1 \%$ of all hours in the year. Reducing the peak period should have created enhanced peak effects; however, this change resulted in minimal changes to the results. The subsection below describes how the peak time slice is defined in NEMS and analyzes the results of the findings.

NEMS hourly loads are classified into nine different combinations of seasons (summer, winter, and spring/fall) and times of day (daytime, morning/evening, and night). Vertical blocks representing the average load during each period approximate the hourly loads in each of the nine time slices. This creates a stepwise approximation to the load duration curve (LDC). The two seasonal/time-of-day groups containing the summer and winter peak loads are further separated into peak and off-peak segments, making a total of 11 categories.

The size of the summer peak segment is controlled by input in the ldsmstr file, which represents the fraction of peak hours in relation to all summer midday hours. In other words, the input data specifies the widths of the steps in the LDC approximation. The default percentage of summer peak hours relative to the total number of summer midday hours is $5 \%$ or approximately 60 hours out of the approximate 1220 summer midday hours, where summer is comprised of the months from June to September. The peak hour percentage was changed first to 1\% ( 12 hours) and then to $0.1 \%$ ( $\sim 1$ hour) to see whether minimizing the number of system peak hours would increase the system peak. However, the difference in peak impacts was minimal as a result of these modifications. The reason for this is likely that this exercise was performed using the default AEO2001 space-cooling load shapes, which peak in October so the residential spacecooling end use peak is not coincident with the system peak. In other words, as long as NEMS represents space-cooling using October-peaking load shapes, it is not possible to determine the impact of CAC standards on peak demand.

\subsection{The Wrong Load Shape}

Berkeley Lab also investigated how the load shapes are input to NEMS-BRS. It was learned that the October-peaking load shape used for the residential space-cooling end use does not accurately represent typical space-cooling loads. As a result of reporting this finding to EIA, a number of loadshapes were updated in the AEO2002 version of NEMS. The subsection below details this finding and EIA's response to Berkeley Lab's request to correct the space-cooling end-use load shape.

For much of the investigation, it was assumed that the RSFCAO67 coded load profile represented the space-cooling end use in NEMS. Although this load shape does correspond to a residential CAC end-use load profile, it is not the load shape that NEMS uses. In-depth investigation of the ldsmstr structure input file pointed to a default load profile called RSFSCE67 
that actually represents the residential space-cooling end use. This load profile was found to peak in October, which is counter to all evidence that space-cooling use is highest in mid to late summer when the weather is generally hottest.

This finding helped explain why modeling was producing such a poor peak demand response to proposed CAC standards. When Berkeley Lab substituted load shape RSFCAO67 into NEMS, a more representative load shape for space cooling which has a summer peak, the impact of CAC standards on system peak was more consistent with expectation. With this substitution, the TSL 4 case CLF declined from approximately $46 \%$ to $25 \%$ using the 2001 version of NEMS-BRS. If the squelch is removed together with the RSFCAO67 load shape in place, the CLF drops a further to $12 \%$ under the SEER 13 standard.

Table 2 below summarizes the differences in CLF resulting from switching the end-use load shape from one that has an inherent CLF of 9\% (RSFSCE67) going into the model to one whose CLF is only 6\% (RSFCAO67). Clearly, the load-shape change has a significant effect on how NEMS-BRS represents to this peaky end use. Changing this end-use load shape resulted in a 3035 point reduction in the CLF overall.

Table 2. Comparing the CLF Using 2 Different CAC Load Shapes Under the APS Customary Energy Decrement Approach in AEO2000 and AEO2001

\begin{tabular}{|c|c|c|c|c|}
\hline & \multicolumn{2}{|c|}{ AEO2000 } & \multicolumn{2}{c|}{ AEO2001 } \\
\hline & RSFSCE67 & RSFCAO67 & RSFSCE67 & RSFCAO67 \\
\hline TSL 1 & $43 \%$ & $30 \%$ & N/A & $27 \%$ \\
\hline TSL 2 & $36 \%$ & $24 \%$ & N/A & $26 \%$ \\
\hline TSL 3 & $36 \%$ & $24 \%$ & N/A & $26 \%$ \\
\hline TSL 4 & $35 \%$ & $23 \%$ & $46 \%$ & $25 \%$ \\
\hline TSL 5 & $41 \%$ & $28 \%$ & N/A & $27 \%$ \\
\hline Roll-Up TSL2 & $36 \%$ & $21 \%$ & N/A & $24 \%$ \\
\hline Roll-Up TSL4 & N/A & $23 \%$ & N/A & $25 \%$ \\
\hline
\end{tabular}

Berkeley Lab notified EIA in August 2001 of this finding regarding the load shapes in NEMS. Shortly thereafter, a number of load shapes in the residential, commercial, and industrial sectors 
were updated for the AEO2002 version of NEMS. ${ }^{3}$ John Holte of OnLocation, Inc. was subcontracted to investigate the NEMS load shapes and make updates in response to the finding.

Residential sector updates were made in the AEO2002 version of NEMS to the load shapes for space heating, space cooling, water heating, cooking, and refrigeration, among others. In addition, residential load shapes were expanded to represent each of the 13 NERC regions, in place of the single load shape that had previously been used to represent the entire U.S. Cooling degree-day data from the Short Term Energy Outlook were used to produce regional load profiles. For space cooling, the new and improved load shape was a mix of the following end uses: CAC (41.3\%), heat pumps for cooling (41.3\%), and room AC $(17.3 \%)$.

\footnotetext{
${ }^{3}$ Matt Lackey of EIA provided a partial copy of a memo written by OnLocation explaining the load shape updates in October of 2001. For more information on this write up, please refer to Alternative Sectoral Load Shapes for NEMS by OnLocation, August 2001.
} 



\section{Proposed Method}

This section of the memo discusses some of the alternative approaches Berkeley Lab tried during the past two years to improve NEMS's forecasted peak impacts of a proposed CAC standard. Ultimately, the traditional end-use decrement approach using the AEO2002 updated load shapes was determined to be the most defensible approach to performing utility and environmental analyses for the $\mathrm{CAC}$ rulemaking. Before making this determination, the following methodologies were considered:

- Efficiency-Modifying approach - The forecasted CAC efficiency level in NEMS is changed to model the proposed CAC standard SEER level.

- The 18-point Approach - This approach uses all higher-decrement CAC runs from all TSLs analyzed in a CAC analysis to derive each standard's utility and environmental impacts, rather than using only the limited number of higher-decrement runs that correspond to a particular TSL, which had been Berkeley Lab's customary strategy.

- The AEO2002 Load Shapes Approach - Newly updated AEO2002 load shapes were used in the AEO2001 analysis.

Each of these techniques is described in the following subsections, with comparison of each technique's degree of improvement relative to the traditional end-use decrement approach using the nonupdated NEMS default load shapes.

\subsection{The Efficiency-Modifying Approach}

The first alternative Berkeley Lab tried in response to a suggestion from EIA after initial CAC peak demand response issues were raised was to manipulate the CAC end-use efficiency level. The residential CAC SEER efficiency level was modified to mimic the benefits to a proposed CAC TSL. Initially, this approach made sense because it so closely matched the objective of modeling forecasted improvements in CAC efficiency levels. Although this approach improved the CLF results, it was difficult to replicate in subsequent versions of NEMS, which made it difficult to use given the tight time constraints associated with the rulemaking process.

For this approach, the rtekty residential technology characteristics NEMS-BRS input file was modified to forecast higher efficiency CAC options to simulate the energy savings from this end use. This method modifies the residential technology performance, allowing only one CAC efficiency or SEER level option during the years of the proposed standard, 2006-2020. Just as in the interpolation methodology used in the conventional approach, three higher-efficiency SEER levels, SEER 24, 35 and 45 were selected to represent the three higher decrement NEMS-BRS runs for each CAC standard to ensure that the results would be seen in the context of the entire energy sector. Although CAC and heat pumps are treated as one rulemaking, CAC and $\mathrm{HP}$ cooling energy savings were modeled using the rtekty method and the heating load from heat pumps used the decrement approach. 
As a result of the rtekty approach, CLF results increased the peak impacts. For example, the CAC TSL 4 CLF dropped from 35\% to $27 \%$ (Table 3) with this alternative method. This is equivalent to $3.5 \mathrm{GW}$ more cumulative installed generating capacity by 2020 for this TSL. But despite the improvements with the results, it raised some concerns. This approach is an improvement to the CLF of $35 \%$ previously mentioned in this memo, as it is closer to the national average of $15 \%$ for CAC use. ${ }^{4}$

One concern about using this method with NEMS-BRS was that the National Energy Savings (NES) model, the source of energy decrement inputs to NEMS-BRS, was less optimistic than NEMS-BRS in predicting the shipment-weighted SEER level for CAC. The NES model is the source for the energy savings input to NEMS-BRS; NEMS presumes an average shipment weighted SEER of 10.7 through 2020 and beyond. The NEMS Reference Case shipment weighted average was a SEER 12.5 for the AEO2001 version. This difference in assumed efficiencies raised concerns at DOE. The main concern was that the higher CAC efficiency level in NEMS could impact the associated costs over the forecast period and produce different results than if the assumptions matched. Furthermore, this approach was difficult to replicate from year to year because of changes to the Reference Case.

It was unclear why this approach lowered the CLF, because simply modifying the efficiency level of the $\mathrm{CAC}$ end use did not seem to be any more or less accurate than using decrements to energy consumption. Thus, this approach was abandoned because it was less defensible than the end-use decrement approach.

\subsection{The 18-point Approach}

Another approach Berkeley Lab considered involved using a complete set of the 18 higher decrement runs from the various standards. This process entailed fitting a linear regression through all 18-points constituting a complete CAC analysis rather than just using the three higher-decrement runs as was the practice in the usual approach. Using 18 points versus three could provide a greater distribution of data points and thereby reveal a more accurate regression than would result from the traditional approach.

This process, unfortunately, resulted in very poor correlations, far worse than those derived using the traditional three-point method. The 18-point approach assumed a linear fit between the change in coal capacity and other fossil fuel capacity with change in residential sales (TWh energy savings). However, the constraint of having to force this line through zero resulted in a very poor regression fit, e.g., $\mathrm{R}^{2}=5 \%$ for Other Fossil Capacity. The line was forced through zero to indicate that zero energy savings imply zero change in capacity. Figure 2 below illustrates what happened to Other Fossil Capacity in 2020 using the AEO2000 version of NEMS (and including the AEO2002 load shapes). However, although for varying changes in residential electricity sales, the change in fossil capacity shows a reasonably clear linear relationship and the 18 points alone fit a highly correlated line, the added constraint of forcing the line through zero significantly shifted the linear trend. A similar pattern was seen in the AEO2001 results. Because of this poor regression fit, the results using this approach were discredited. Berkeley

\footnotetext{
4 Taken from a paper by Koomey, Rosenfeld and Gadgil, 1990. "Conservation Screening Curvest to Compare Efficiency to Power Plants.” Paper from Energy Policy, Volume 18 No. 8, October 1990.
} 
Lab therefore decided not to use this method because the regression fit was so poor and the interpolated GW savings were not reliable given the low $\mathrm{R}^{2}$ values.

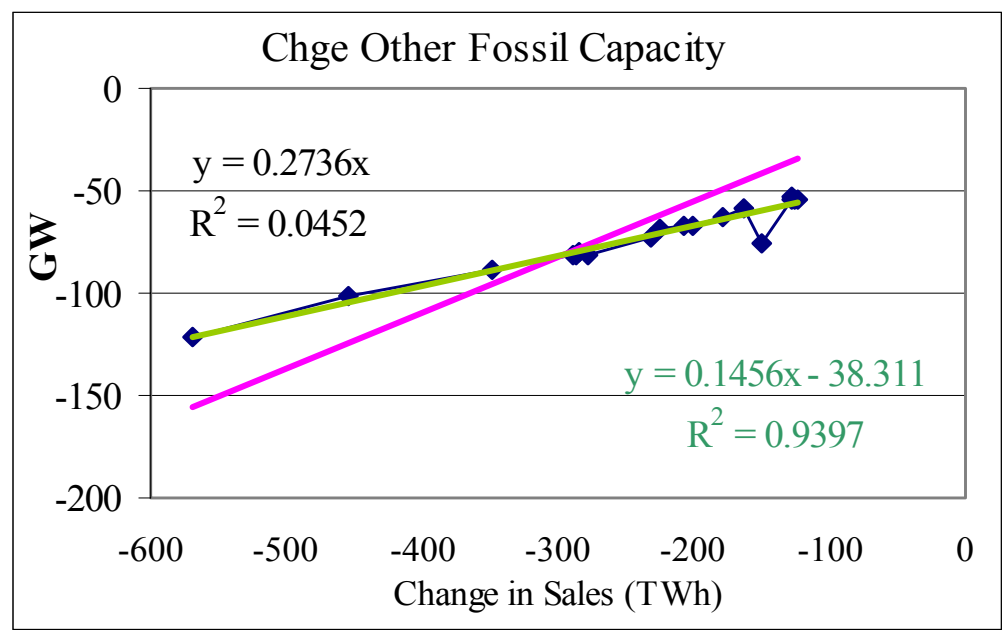

Figure 2. Change in Other Fossil Capacity for 18 Runs

For NOx marginal emissions factors, Berkeley Lab also debated changing the methodological approach to a similar linear regression of the 18 points from each of the higher-decrement runs. Figure 3 below shows the plot of the 18 runs' NOx marginal emissions factors (MEFs) in the year 2014. The initial theory was that each set of 18 points (one set per forecast year) might produce a strong linear trend. However, when plotted, some of the earlier years show no consistent relationship between the NOx MEF and the change in sales. Rather, the graph looked more like a scatter plot. After further scrutiny, it became apparent that simply taking the average of the three points as had previously been done (rather than trying to fit a linear trend) was the best approach for this analysis. Figure 3 shows that enlarging the y-axis of the plot reveals a much flatter trend, which supports the use of a simple average approach to treat marginal emissions factors. In sum, the 18-point method was decidedly unsuccessful at improving the peak impacts for the CAC analysis. 


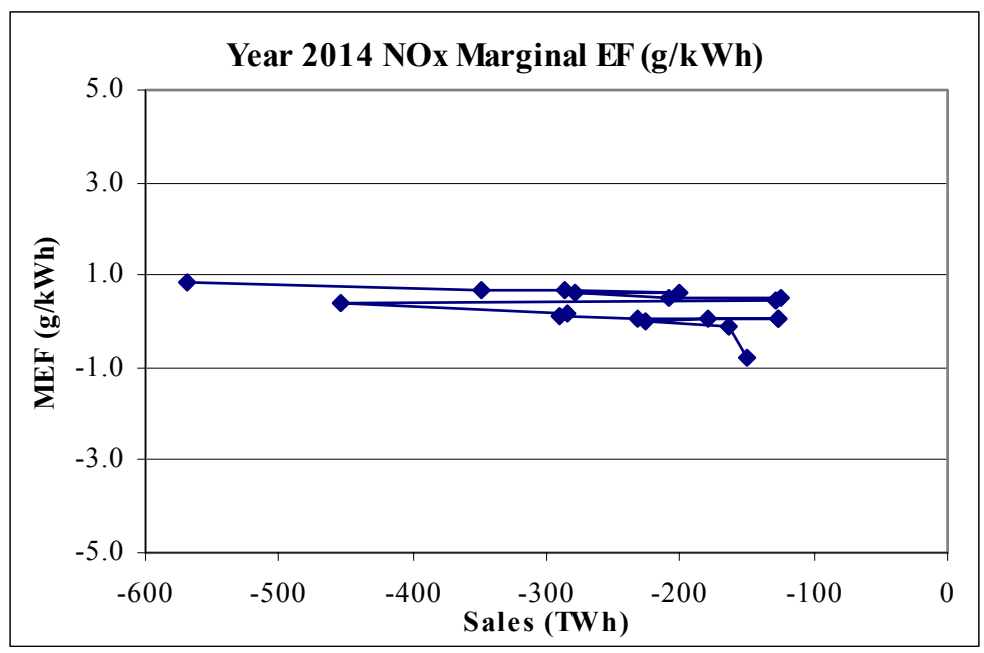

Figure 3. NOx Marginal Emissions Factors from 18 Runs (2015)

\subsection{Using the AEO2002 Load Shapes}

Following EIA's update of the load shapes in the AEO2002 version of NEMS, Berkeley Lab experimented with using these new load shapes to determine their impact on peak savings from proposed CAC standards. After a series of experimental test runs, Berkeley Lab determined that this approach is the most defensible way to perform the CAC analysis. This section describes the changes that were made to replace the load shapes and the differences in the results using the new and default load shapes. Appendix B explains step by step how this approach was performed.

Berkeley Lab began experimenting with the AEO2002 version of the NEMS load shapes when it first became available in December 2001. A series of runs was performed using the 2002 load shapes in previous versions of the NEMS code (AEO2000 and AEO2001). ${ }^{5}$ The purpose of this exercise was to determine the effect of the improved load shapes.

Berkeley Lab performed a full set of proposed CAC standards runs using the AEO2002 load shapes in the AEO2001 source code to determine the impacts on peak reduction. This exercise superseded the runs replacing the RSFSCE67 with the RSFCAO67 load shape (described in Section 4.4.) Analysis using the 2002 load shapes was performed using the old method of decrementing the end-use consumption for each of the standards, and by using the AEO2002 load shapes ${ }^{6}$ by replacing the $l d s m s t r^{7}$ and $l d s m d a f$ input files. Generally, this analysis resulted in CLFs that ranged from $29 \%$ to $33 \%$ for the various TSLs and peak impacts that were higher than those calculated using the default load shapes (e.g., $12.7 \mathrm{GW}$ vs. $9.0 \mathrm{GW}$ in avoided capacity in 2020 for TSL 4 using the 2001 version of NEMS-BRS and the 2002 load shapes). Shortly after this analysis was completed, DOE requested a repeat analysis with the AEO2000 version of

\footnotetext{
${ }^{5}$ Older versions of NEMS were used because the AEO2002 version of NEMS was not fully functional on our machines.

${ }^{6}$ This analysis is saved on Kristina Hamachi LaCommare's computer in:

D:\Appliances $\backslash$ aeo2001\CAC.HP_Oct.2001 \with_AEO2002_ldsmdaf.ldsmstr.

${ }^{7}$ The ldsmstr file is a text structure file that assigns the various end uses to a load profile that is contained in the binary, ldsmdaf, data file.
} 
NEMS so that the results would be easy to compare with the published CAC analysis using the AEO2000 version and the rtekty approach.

A comparable analysis was done with the AEO2000 version of NEMS-BRS. The peak impacts for the AEO2000 analysis using the 2002 load shapes were slightly higher than those yielded by the AEO2001 version (12.8 GW vs. $12.7 \mathrm{GW}$ for TSL 4 in 2020). The CLF ranged from $31 \%$ to $37 \%$, slightly less reasonable than the results from the AEO2001 analysis, yet an improvement over the AEO2001 CLFs (approximately 46\% for TSL 4) using the traditional approach. However, this approach resulted in lower peak impacts compared to the approach of using the rtekty input file to manipulate the CAC efficiency levels (15.5 GW in 2020).

Table 3 below compares some of the approaches Berkeley Lab used to try and improve and better understand the results. In general, the approach using the 2001 version of NEMS-BRS resulted in slightly lower peak impacts than were found using the 2000 version. This was largely due to updates to the Reference Case version of NEMS from 2000 to 2001. The end-use decrement approach yielded the worst CLFs of all the approaches considered. Modifying the efficiency level of the CAC end use improved the CLF to $27 \%$ from $35 \%$ using the 2000 version of NEMS-BRS. Adding the switch to the RSFCAO67 load shape further reduced the CLF to $14 \%$ in 2020 . Just using the traditional approach with the switched load shape yielded a CLF of 23\%; when AEO2002 load shapes were used, a CLF of 33\% CLF was calculated in the 2000 version of NEMS-BRS.

Table 3. Comparing 2020 CLFs from Various Approaches Using TSL 4

\begin{tabular}{|l|c|c|}
\hline Approaches for SEER 13 (Standard 4) & AEO2000 & AEO2001 \\
\hline Traditional End-use Decrement Approach & $35 \%$ & $46 \%$ \\
\hline Rtekty Approach with Default Load Shapes & $27 \%$ & N/A \\
\hline Rtekty Approach with CAO Load Shape & $14 \%$ & N/A \\
\hline Traditional End-Use Decrement Approach with CAO Load Shape & $23 \%$ & $25 \%$ \\
\hline Traditional End-Use Decrement Approach with AEO2002 Load Shapes & $33 \%$ & $33 \%$ \\
\hline
\end{tabular}

Ultimately, the published CAC analysis was performed using the 2002 load shapes with the enduse decrement approach and the standard three-point interpolation for utility impacts and the three-point average for the MEFs in the 2000 version of NEMS-BRS. The peak impacts increased slightly from $11.7 \mathrm{GW}$ to $12.8 \mathrm{GW}$ for TSL 4 in 2020 with this approach. However, compared to the previously published version of the analysis (using the rtekty approach and default load shape), this work resulted in 17\% less capacity savings in 2020. Even though the traditional approach using the 2002 load shapes resulted in higher CLFs than the other tested approaches, the modified approach was chosen because it applies the most credible analysis approach. 



\section{Conclusions and Future Work}

Berkeley Lab's analysis of the treatment of residential CAC load shapes in NEMS-BRS uncovered some problems that help explain the puzzling small peak impacts found in initial analysis of proposed CAC standards.

Our key findings were:

- NEMS was using an inappropriate load shape to represent residential space cooling. Berkeley Lab found that the space-cooling end use was represented in the 2001 (or earlier) version of NEMS by an October-peaking load profile, not a more representative one like the RSFCAO67 load shape included in inputs but not activated. The CLF dropped from $35 \%$ to $23 \%$ for proposed CAC standards when replacing the autumn-peaking load shape with the summer-peaking RSFCAO67. Subsequent to this finding, EIA responded by updating a number of end-use load shapes to NEMS, including the residential space cooling load shape.

- NEMS uses a squelch factor to scale down system load so that it coincides with the historical data used to initialize the model. Removing this squelch has a dramatic effect on peak impacts -- a 3.5\% increase in the AEO2001 Reference Case installed capacity in 2020. Note that the squelch lowers all NEMS peak load growth forecasts. The presence of the squelch in NEMS helps explain why prior peak-load-reduction results are lower than expected.

- Using the updated AEO2002 load shapes with the customary end-use decrement approach was the approach used for the CAC rulemaking analysis. An EIA-approved approach for conducting this analysis was chosen rather than other "fixes" that hard wired the results in keeping with expectations but don't logically represent the end use better. This chosen approach improves the CLF from the AEO2001 analysis from 45\% to $33 \%$.

Based on the efforts discussed in this report, Berkeley Lab now has a good understanding of why the initial CAC peak impacts from NEMS were less then expected. The discovery of both the inappropriate space-cooling load shape in the 2001 version of NEMS-BRS and the squelch factor explain the troublesome results. EIA responded in a timely manner to Berkeley Lab's request to refine the NEMS load shapes. Although improving the load shapes improved the CLF (from $45 \%$ to $33 \%$ for TSL 4 ), the presence of the squelch still appears to be dampening peak impacts. The findings further show that the customary end-use decrement approach is valid. This is particularly important because two upcoming rulemakings cover peak coincident loads, namely commercial air conditioning and distribution transformers.

This analysis raises opportunities for some interesting future work. With the detailed understanding Berkeley Lab now has of how the load demand is structured in NEMS, better modeling of this submodule to more realistically treat the impacts on the annual load would be worthwhile. This would involve evaluating both Delta approaches to determine which one is most effective and more closely analyzing the squelch and its role in the model. Learning more about how well some of the other end uses like furnaces, boilers or commercial CAC are represented in NEMS as it pertains to future rulemakings would also be advantageous. 



\section{Appendix A: Mathematical Representation of Load Demand in NEMS}

This appendix describes the mathematical differences between these two approaches and presents some possible reasons why the Original Delta Approach was deactivated in the model.

The mathematical representation of the Original Delta Approach is shown in the documentation as:

$$
\begin{aligned}
& \operatorname{SYLOAD}(\mathrm{H})=\operatorname{DistLo}_{\mathrm{s}}(\mathrm{H}) * \operatorname{SysLoad}_{\mathrm{BaseYr}_{\mathrm{r}}}+\Sigma \operatorname{DistLo}_{\mathrm{e}}(\mathrm{H}) *\left(\operatorname{load}_{\mathrm{e}}^{\mathrm{yr}}-\operatorname{load}_{\mathrm{e}}^{\text {BaseYr }}\right) \\
& =\Sigma \operatorname{DistLo}_{e}(\mathrm{H}) * \operatorname{load}_{e}^{\mathrm{yr}}+\left(\operatorname{DistLo}_{\mathrm{s}}(\mathrm{H}) * \operatorname{SysLoad}_{\mathrm{Base}_{\mathrm{Yr}}}-\Sigma \operatorname{DistLo}_{\mathrm{e}}(\mathrm{H}) * \operatorname{load}_{\mathrm{e}}{ }^{\text {BaseYr }}\right) \\
& =\sum \operatorname{DistLo}_{\mathrm{e}}(\mathrm{H}) * \operatorname{load}_{\mathrm{e}}^{\mathrm{yr}}+\operatorname{SysLoad}_{\mathrm{BaseYr}} *\left(\operatorname{DistLo}_{\mathrm{s}}(\mathrm{H})-1 / \operatorname{SysLoad}_{\mathrm{BaseYr}} * \sum \operatorname{DistLo}_{\mathrm{e}}(\mathrm{H}) *\right.
\end{aligned}
$$

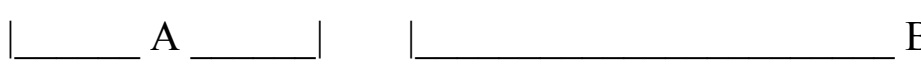

where

$\Sigma \quad=\quad$ sum over all end uses, e

BaseYr $=\quad$ base year (set in the model to 2001 in AEO2001 version of NEMS-BRS)

$\operatorname{SYLOAD}(\mathrm{H})=\quad$ system load in hour, $\mathrm{H}$

$\operatorname{DistLo}_{\mathrm{s}}(\mathrm{H})=$ historical hourly load shape for the system

$\operatorname{DistLo}_{\mathrm{e}}(\mathrm{H})=$ hourly load shape for end use, e

SysLoad $_{\text {BaseYr }}=\quad$ total system load in base year (2001 in AEO2001 version of NEMS-BRS)

$\operatorname{load}_{\mathrm{e}}^{\mathrm{yr}} \quad=\quad$ current year load for end use, $\mathrm{e}$

$\operatorname{load}_{\mathrm{e}}{ }^{1990}=\quad$ base year (2001 in AEO2001 version of NEMS-BRS) load for end use, $\mathrm{e}$

Note: Term $\mathrm{B}$ is a constant for each hour $\mathrm{H}$, so a different constant term for each hour is added to the hourly total system load shape represented by term A. When comparing the Reference Case system load shape and the higher-decrement-case system load shape, the difference in the hourly load will cancel out the term B.

The current Delta Approach is shown below; its representation of the system load is similar to that of the original Delta Approach:

$\operatorname{SYLOAD}_{(H)}=\operatorname{DistLo}_{\mathrm{s}}(\mathrm{H}) * \operatorname{SysLoad}_{\mathrm{yr}}+\Sigma \operatorname{DistLo}_{\mathrm{e}}(\mathrm{H}) *\left(\operatorname{load}_{\mathrm{e}}^{\mathrm{yr}}-\operatorname{SysLoad}_{\mathrm{yr}} / \operatorname{SysLoad}_{\mathrm{BaseY}} *\right.$ $\operatorname{load}_{\mathrm{e}}{ }^{\text {BaseYr}}$ )

$$
=\Sigma \operatorname{DistLo}_{\mathrm{e}}(\mathrm{H}) * \operatorname{load}_{\mathrm{e}}^{\mathrm{yr}}+\left(\operatorname{DistLo}_{\mathrm{s}}(\mathrm{H}) * \operatorname{SysLoad}_{\mathrm{yr}}-\operatorname{SysLoad}_{\mathrm{yr}} / \operatorname{SysLoad}_{\mathrm{BaseYr}} * \Sigma\right.
$$

$\operatorname{DistLo}_{\mathrm{e}}(\mathrm{H}) * \operatorname{load}_{\mathrm{e}}$ BaseYr $^{*}$

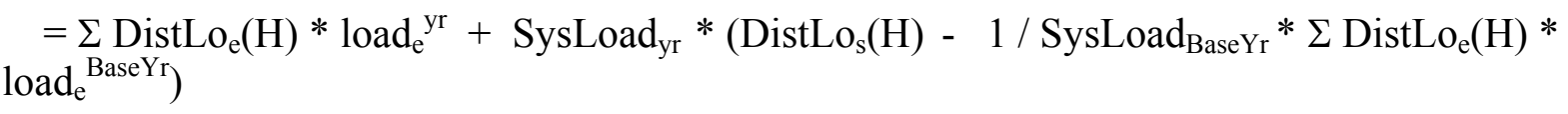

A

B 
Investigation of Residential Central Air Conditioning Load Shapes in NEMS

where SysLoad $\mathrm{yr}_{\mathrm{r}}=\sum \operatorname{load}_{\mathrm{e}}^{\mathrm{yr}}=$ total system load in current year

In this formulation, the only difference from the original equation is term B where SysLoad yr $_{\text {is }}$ used as a scaling factor instead of SysLoad ${ }_{\text {BaseYr }}$, so term B varies by year after $2001 .^{8}$

\footnotetext{
${ }^{8}$ In the NEMS coding, this annually varying system load factor, $s l r$, is located on line 5,276 of the uldsm. $f$ sub-module. This piece of coding is located in the subroutine DSMDLT, in the LDSM submodule, which is responsible for performing the Delta Approach.
} 


\section{Appendix B: Detailing the Traditional End-Use Decrement Approach Using the 2002 Load Shapes}

This approach was performed as follows:

1. A decrement was made to the end-use consumption (e.g., residential space cooling) by adding an input file into NEMS-BRS called savings.dat. This file contained the site MWh annual energy savings for each end use. The size of the decrement was determined to be some higher multiplier of the actual standard savings to be seen in the context of the large energy sector.

2. An input file called $c d$ mult.dat was also added to partition the savings regionally based on consumption usage from the Residential Energy Consumption Survey (RECS).

3. Code changes were applied to the residential module to read in the savings.dat and $c d$ mult.dat files and to decrement the space-cooling end-use consumption.

4. The AEO2001/2000 ldsmdaf input file was replaced with the AEO2002 version, which contains the updated load profiles for numerous end uses in the residential, commercial, and industrial sectors.

5. The AEO2001/2000 ldsmstr input file was replaced with the AEO2002 version, which points each end use to an hourly load profile in the ldsmdaf file.

6. The FILELIST was modified to point to the correct ldsmdaf and ldsmstr files.

7. Three higher-decrement NEMS-BRS runs were performed for each proposed residential CAC standard.

8. A linear regression on the three higher-decrement runs was used to derive the utility sector impacts on generation and installed generating capacity for each fuel type and each forecasted year via interpolation. 